\title{
High-fidelity, High-speed, 3-D Finite-element Modeling of Acoustic Scattering from Objects in Shallow Water
}

\author{
Dr. David S. Burnett \\ Code HS11 \\ Naval Surface Warfare Center \\ 110 Vernon Ave. \\ Panama City, FL 32407-7001 \\ phone: (850) 235-5332 fax: (850) 234-4888 email: david.s.burnett@navy.mil \\ Document Numbers: N0001408WX20233 ; N0001408WX20775 ; N0001408WX20126 \\ http://www.ncsc.navy.mil
}

\section{LONG-TERM GOALS}

The goal is to bring to fruition R\&D that has been performed for ONR since FY05. The work has involved the development of a state-of-the-art, high-fidelity, high-speed, 3-D finite-element (FE) broadband computer simulation capability for modeling the scattering of sonar signals by undersea objects located in or near the seabed in littoral environments with smooth or rippled water/sediment interfaces (Fig. 1). Many of the computational techniques developed in this effort will be relevant to future ONR programs involving different applications.

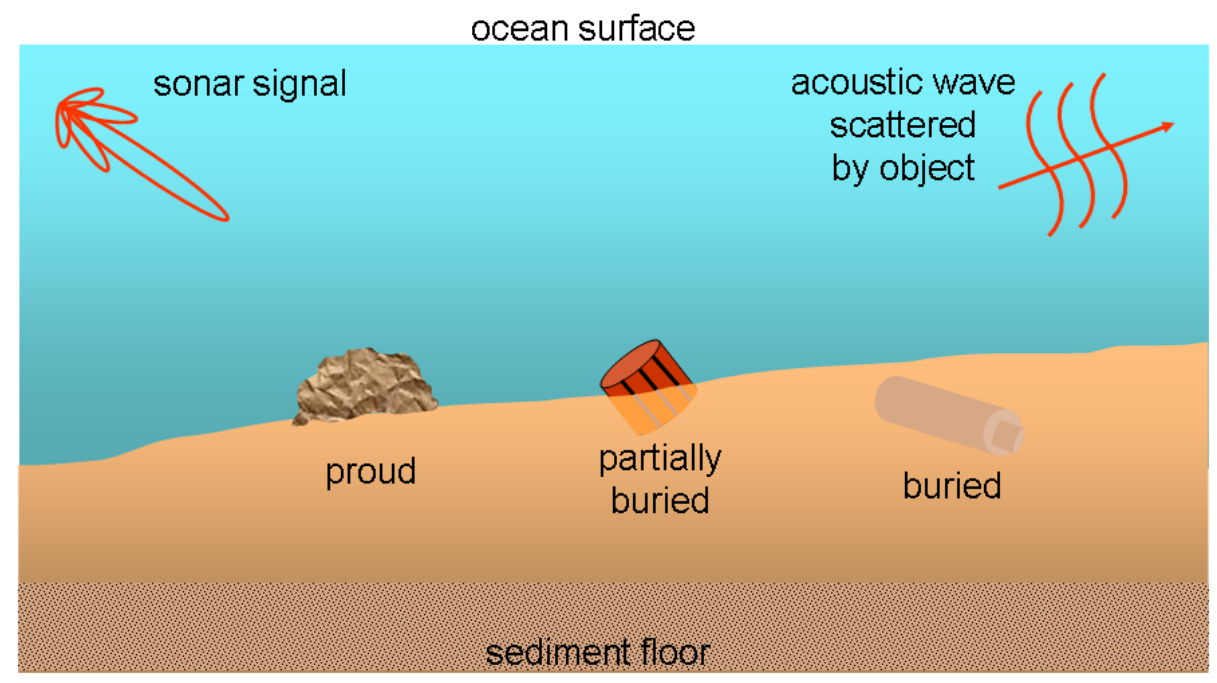

Figure 1. A representative shallow-water acoustic scattering scenario.

\section{OBJECTIVES}

The primary objective is to provide the U.S. Navy with a high-fidelity, high-speed computer simulation tool for computing and displaying the frequency and aspect angle response (a.k.a. "acoustic color") of complicated, realistic objects in littoral environments. A secondary objective is to maintain a 


\section{Report Documentation Page}

Form Approved

OMB No. 0704-0188

Public reporting burden for the collection of information is estimated to average 1 hour per response, including the time for reviewing instructions, searching existing data sources, gathering and maintaining the data needed, and completing and reviewing the collection of information. Send comments regarding this burden estimate or any other aspect of this collection of information,

including suggestions for reducing this burden, to Washington Headquarters Services, Directorate for Information Operations and Reports, 1215 Jefferson Davis Highway, Suite 1204, Arlington

VA 22202-4302. Respondents should be aware that notwithstanding any other provision of law, no person shall be subject to a penalty for failing to comply with a collection of information if it

does not display a currently valid OMB control number.

\begin{tabular}{|c|c|c|}
\hline $\begin{array}{l}\text { 1. REPORT DATE } \\
\mathbf{3 0} \text { SEP } 2008\end{array}$ & $\begin{array}{l}\text { 2. REPORT TYPE } \\
\text { Annual }\end{array}$ & $\begin{array}{l}\text { 3. DATES COVERED } \\
\mathbf{0 0 - 0 0 - 2 0 0 8} \text { to 00-00-2008 }\end{array}$ \\
\hline \multirow{3}{*}{\multicolumn{2}{|c|}{$\begin{array}{l}\text { 4. TITLE AND SUBTITLE } \\
\text { High-fidelity, High-speed, 3-D Finite-Element Modeling Of Acoustic } \\
\text { Scattering From Objects In Shallow Water }\end{array}$}} & 5a. CONTRACT NUMBER \\
\hline & & 5b. GRANT NUMBER \\
\hline & & 5c. PROGRAM ELEMENT NUMBER \\
\hline \multirow{3}{*}{\multicolumn{2}{|c|}{ 6. AUTHOR(S) }} & 5d. PROJECT NUMBER \\
\hline & & 5e. TASK NUMBER \\
\hline & & 5f. WORK UNIT NUMBER \\
\hline \multicolumn{2}{|c|}{$\begin{array}{l}\text { 7. PERFORMING ORGANIZATION NAME(S) AND ADDRESS(ES) } \\
\text { Naval Surface Warfare Center,110 Vernon Ave.,Panama } \\
\text { City,FL,32407-7001 }\end{array}$} & $\begin{array}{l}\text { 8. PERFORMING ORGANIZATION } \\
\text { REPORT NUMBER }\end{array}$ \\
\hline \multirow{2}{*}{\multicolumn{2}{|c|}{ 9. SPONSORING/MONITORING AGENCY NAME(S) AND ADDRESS(ES) }} & 10. SPONSOR/MONITOR'S ACRONYM(S) \\
\hline & & $\begin{array}{l}\text { 11. SPONSOR/MONITOR'S REPORT } \\
\text { NUMBER(S) }\end{array}$ \\
\hline
\end{tabular}

12. DISTRIBUTION/AVAILABILITY STATEMENT

Approved for public release; distribution unlimited

13. SUPPLEMENTARY NOTES

code 1 only

14. ABSTRACT

The goal is to bring to fruition R\&D that has been performed for ONR since FY05. The work has involved the development of a state-of-the-art, high-fidelity, high-speed, 3-D finite-element (FE) broadband computer simulation capability for modeling the scattering of sonar signals by undersea objects located in or near the seabed in littoral environments with smooth or rippled water/sediment interfaces (Fig. 1). Many of the computational techniques developed in this effort will be relevant to future ONR programs involving different applications.

15. SUBJECT TERMS

16. SECURITY CLASSIFICATION OF:

a. REPORT

unclassified b. ABSTRACT

unclassified c. THIS PAGE unclassified
17. LIMITATION OF ABSTRACT

Same as Report (SAR)
18. NUMBER OF PAGES

9 19a. NAME OF

RESPONSIBLE PERSON 
continuing R\&D effort in order to significantly increase the computational efficiency of the software, thereby expanding the ability to model more complicated objects at higher frequencies.

\section{APPROACH}

\section{High fidelity}

- Physics: Target scattering models employ fully 3-D physics throughout the computational domain; i.e., the fully 3-D wave equations are used throughout both solids and fluids:

Fluids: linear acoustics (Helmholtz eq.): inviscid, volume dissipation, inhomogeneous

$$
-\nabla \cdot\left(\frac{1}{\omega^{2} \rho} \nabla p\right)-\frac{1}{B} p=0
$$

Solids: linear elasticity (elastodynamics eq.): viscoelastic, anisotropic, inhomogeneous

$$
-\nabla \cdot(c \nabla \boldsymbol{u})-\omega^{2} \rho^{s} \boldsymbol{u}=\boldsymbol{f}^{s}
$$

Equation (2) is used throughout all structural parts of the target, including even thin plates and shells, small fillets and chamfers, etc. This is essential for high fidelity since the physics is strongly 3-D near discontinuities such as shell intersections. Local evanescent elastic waves must be modeled accurately, since their phases and amplitudes determine the propagating elastic waves in the outer casings, which in turn determine the scattered acoustic field launched from the casing.

- Mathematics: Since the infinite family of polynomial basis functions within each finite element spans a complete space, then the FE solution within each element can be arbitrarily close to the exact solution of the physical model.

In summary, all physical phenomena describable by eqs. (1) and (2) are included in the FE solutions.

\section{High speed}

Acoustic color is target strength (TS) as a function of both frequency and aspect angle. To generate an acoustic color plot using computer simulation requires extraordinarily high computational efficiency, since it involves a double sweep: (1) over a broad frequency band, typically several octaves, which usually entails sampling at several hundred frequencies, and (2), for each frequency, a sweep over many aspect angles, again typically several hundred. The double sweep therefore requires $\mathrm{O}\left(10^{5}\right) \mathrm{FE}$ models. In addition, by decomposing the incident field (Fig. 3 under Work Completed) into different types of symmetry components, each model can often be decomposed into 2, 4 or 8 smaller (hence much faster) sub-models. The net result, therefore, can sometimes involve $\mathrm{O}\left(10^{6}\right) \mathrm{FE}$ analyses to produce a single acoustic color plot. For practicality, the goal of this work is to compute an acoustic color plot in about one day, which means $\mathrm{O}\left(10^{6}\right) \mathrm{FE}$ models per day. This goal is close to being accomplished, by a combination of software and hardware development, as described below under Work Completed. 


\section{High reliability}

The control of errors, via verification and validation ("V\&V"), plays a key rôle in this project; it is essential to the goal of providing reliable simulations of the real world. Verification (Fig. 2) refers to the control of errors in the finite-element solution vis-à-vis the exact solution of the idealized mathematical model, i.e., computational/mathematical errors. Validation refers to the control of errors in the exact solution of the idealized mathematical model vis-à-vis the real world, i.e., errors in the physical assumptions and data in the idealized model. V\&V is an ongoing, never-ending exercise as the simulation capabilities mature.

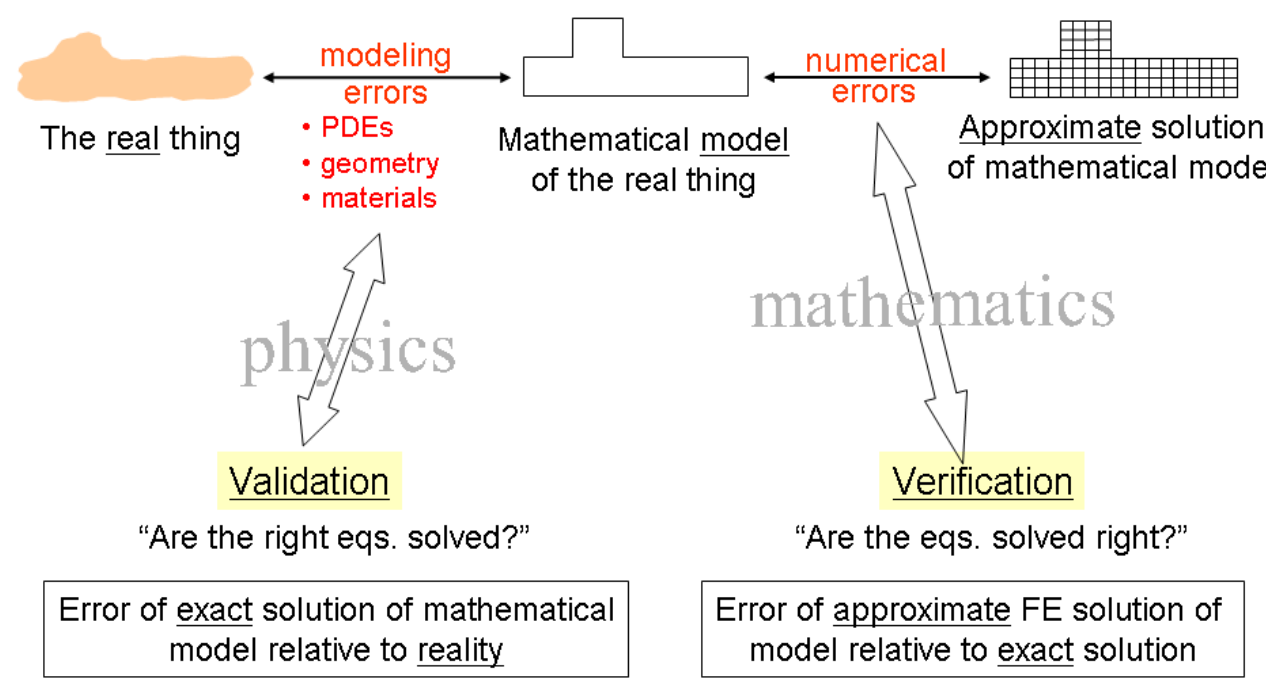

Figure 2. Pictorial explanation of verification and validation.

References [1] and [2] illustrate several verifications. Figure 5 under Results below shows a significant validation milestone: experimental and simulated acoustic color plots exhibiting a striking similarity. $\mathrm{V} \& \mathrm{~V}$ is an ongoing, never-ending exercise as the simulation capabilities mature.

\section{Key individuals participating in this work}

- Leslie H. Wigdor: Syslink Consulting, LLC., 21 Rock Street, Cold Spring, NY 10516-2911. Systems programming; integration of FE and non-FE software; user-interfaces

- Dr. Gary S. Sammelmann: Code HS11, Naval Surface Warfare Center, 110 Vernon Ave., Panama City, FL 32407-7001. Development of non-FE theory and algorithms; analytical solutions to canonical problems for verification.

\section{WORK COMPLETED}

\section{Finite-element R\&D}


As reported in [1] and [2], several modeling techniques were developed during FY06 and FY07:

- New tri-axial ellipsoidal radiation boundary conditions,

- Group-theoretic decomposition of the incident field,

- Parametric sweeping over both frequency and aspect angle,

- Evaluation of scattered field exterior to the FE mesh using Kirchoff-Helmholtz integral.

The motivation for developing all these techniques has been to increase computational efficiency. Each technique has increased computational efficiency by one to two orders of magnitude. The resulting half-a-dozen orders-of-magnitude increase in computational efficiency has resulted in meeting the high-speed requirement of $\mathrm{O}\left(10^{6}\right) \mathrm{FE}$ models per day.

During FY08 these techniques were further refined. However, the major effort was integrating them into an acoustic color system, which is described next.

\section{Software: automated acoustic color system}

Most of the work in FY08 has focused on bringing together all the above-described technologies into a user-friendly GUI-driven software system capable of automatically running $\mathrm{O}\left(10^{6}\right) \mathrm{FE}$ models, managing computer resources and very large amounts of different types of data, and combining the output from all the models into an acoustic color plot, as well as a variety of other plots, e.g., slices of the acoustic color representing frequency response or angular response. The principal functions are listed here, followed by an illustrative plot (Fig. 3) of a scattering problem that is modeled by only one quadrant, due to symmetries, and a sketch of the surface on which Helmholtz integration is performed.

- Sweeping over frequency,

- Sweeping over aspect,

- Management of sub-models, due to symmetries in target and environment; the incident field can be nonsymmetric,

- Dynamic frequency-dependent geometry and meshing to maintain uniform discretization error with frequency - an important feature for signal processing the acoustic color data. (See illutration and explanation under Results below.)

- Computation of Helmholtz integral for TS:

- evaluating near-field $p$ and $d p / d n$ on Helmholtz surface, which includes "virtual" surfaces that don't exist,

- evaluating $G$ and $d G / d n$ for littoral environment,

- GUI:

- Input: parametric ranges, increments, etc.,

- Output: Acoustic color data, in various plots. 


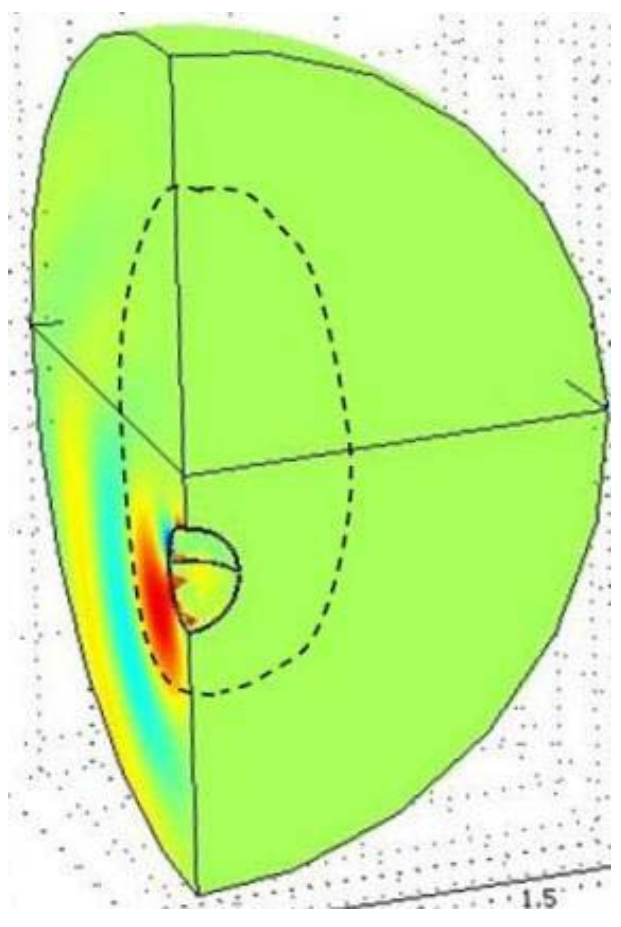

Figure 3. A typical model: one quadrant of a full model of scattering from a buried spherical shell. The dashed contour indicates the edges of a closed surface for Helmholtz integration.

Figure 4 illustrates the user-friendly GUI for controlling a complete acoustic color simulation.

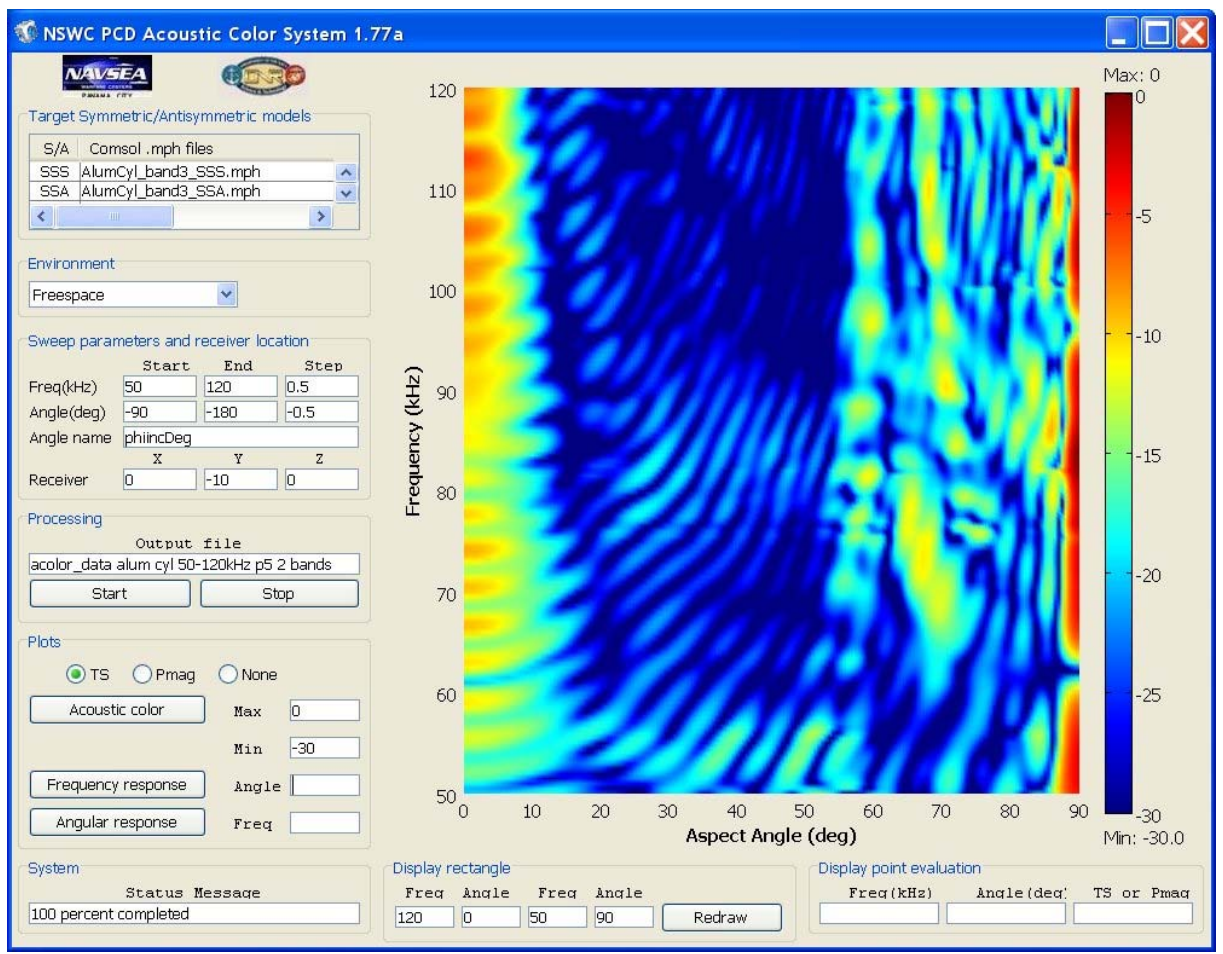

Figure 4. GUI that controls production of acoustic color plots and other output 


\section{Hardware: building a distributed-processing rack-computer system}

As demonstrated under Results below, an acoustic color simulation can typically take a few processordays for "practical accuracy" ( $\sim 1 \mathrm{~dB}$ discretization errors) or as many as several tens of processordays for "high accuracy" ( $\sim 0.1 \mathrm{~dB}$ errors). Distributed processing is therefore necessary to achieve the high-speed requirement of approximately one day to compute an acoustic color data set. An important task during FY08 has been the design, purchase and assembly of a scalable-architecture, multi-blade, parallel-processing rack computer. This is almost finished, including the installation and successful testing of the acoustic color system software. When completely finished (in 1QFY09), the system will have 20 blades, each with two dual-core processors ( $\sim 3$ x speed-up vs one core). Ten blades will have 32 GB RAM (for high frequencies) and ten will have 16 GB RAM (for medium and low frequencies). This combination of blades, RAM and multi-core processors will reduce several tens of processor days to about one day.

In parallel with the hardware build has been the task of modifying the acoustic color system to manage computer resources in a distributed-processing environment, with concomitant modifications to the GUI.

\section{RESULTS}

An important milestone was achieved with the following experimental validation. The acoustic color system was used to model the free-field insonification of a 5-to-1 aspect ratio aluminum cylinder (length 7.5"; diameter 1.5") from 50 to $120 \mathrm{kHz}$ ( $k a$ from 4.2 to 9.7; $k L$ from 42 to 97). The results are compared here (Fig. 5) with the data from a tank experiment performed at Wash. State Univ. [3]. This was a "practical accuracy" simulation, using a relatively coarse sampling in frequency and aspect angle, which required "only" 102,648 FE analyses. A "high accuracy" simulation, using finer sampling, would require $\mathrm{O}\left(10^{6}\right) \mathrm{FE}$ analyses and take twenty to thirty processor days, i.e., about one day on the above-described distributed-processing rack computer. 


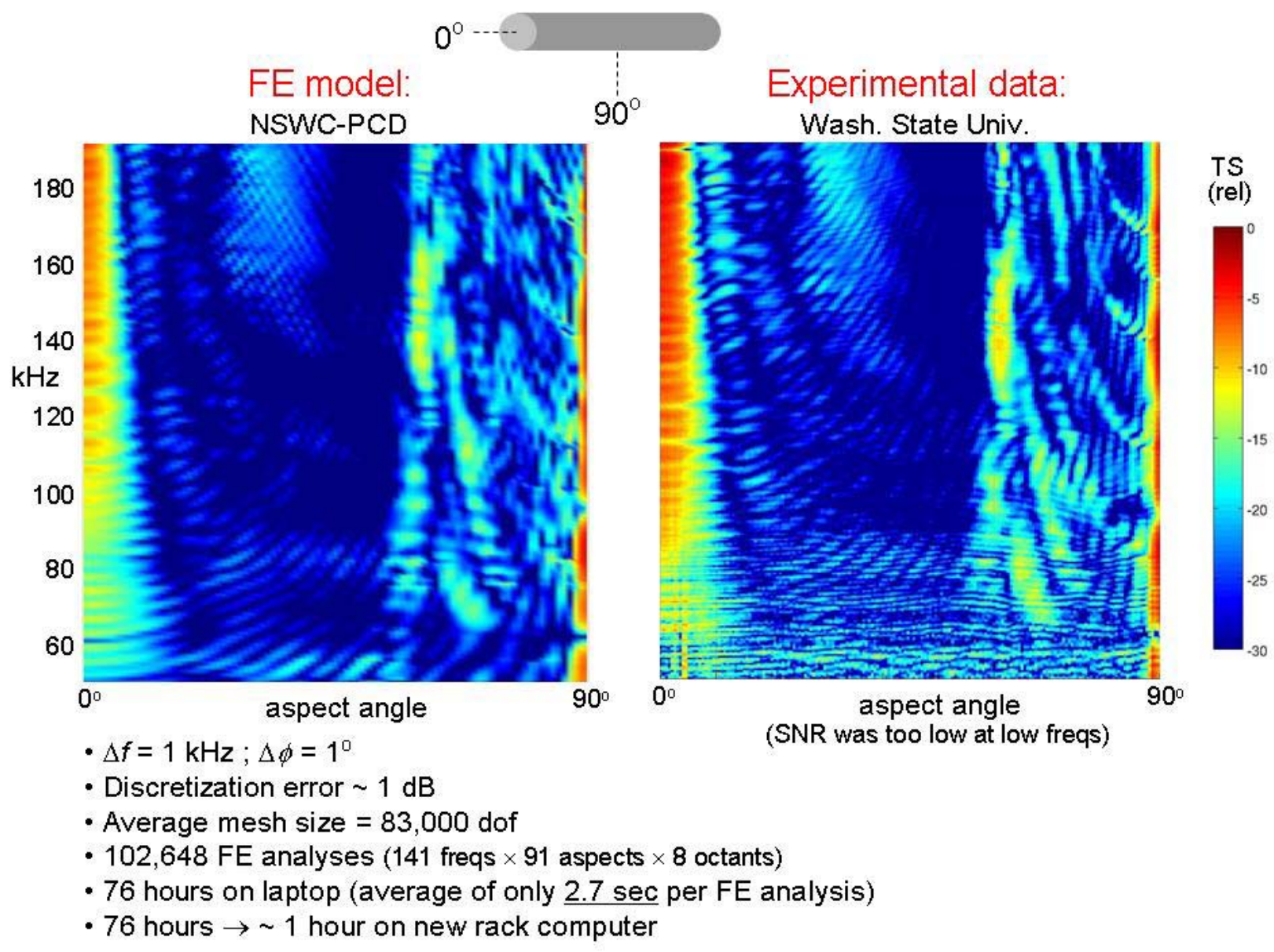

Figure 5. FE model vis-à-vis experimental data

This validation problem illustrates the use of the automated dynamic change to both the outer fluid boundary radius and the mesh density throughout the model. As frequency increases: (1) the outer fluid boundary automatically moves in to maintain a constant-wavelength separation from the target and (2) the mesh is automatically refined to maintain a constant number of elements per wavelength. Figure 6 shows the FE models at three of the 141 frequencies run. The meshes are on one of the faces of the 3-D model in the upper right of the figure, which is one octant of the full physical model. 


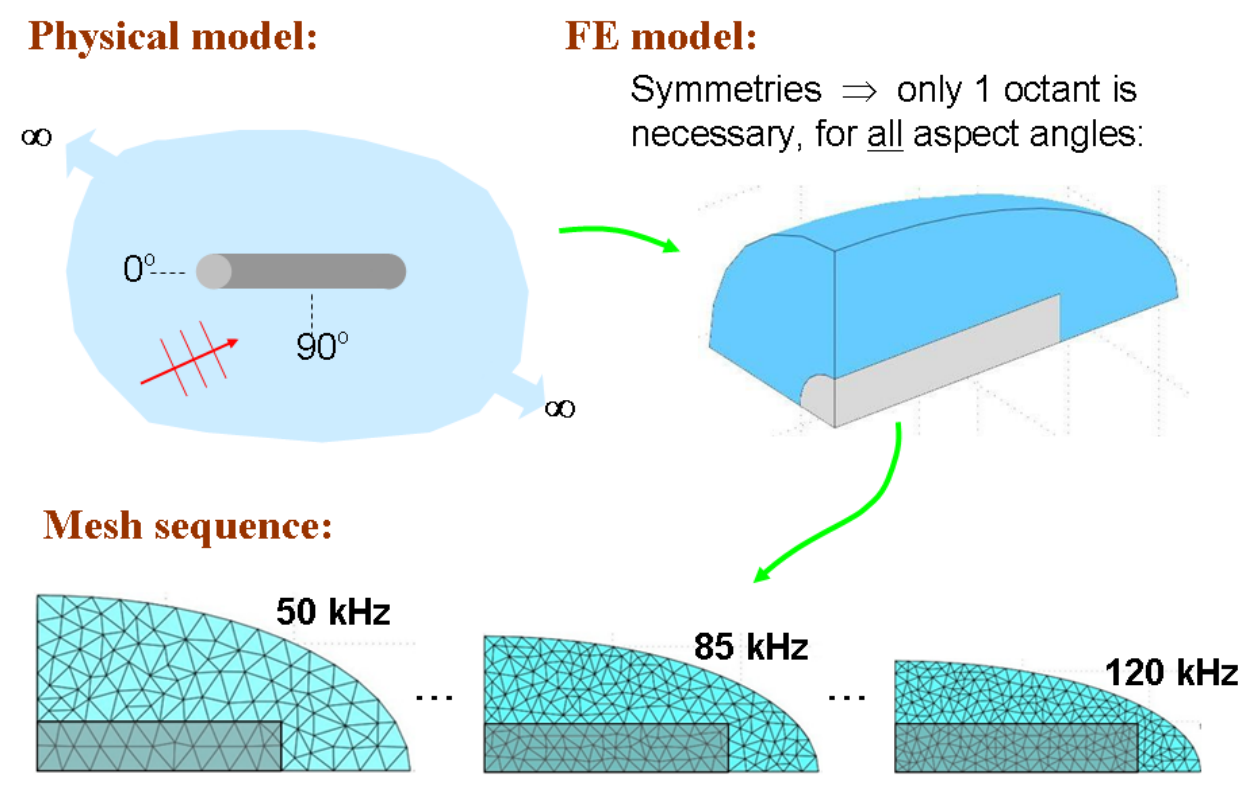

Figure 6. Automated change of geometry and mesh with frequency, showing 3 of the 141 different $F E$ models

\section{IMPACT/APPLICATIONS}

This high-speed, high-fidelity 3-D target scattering simulation capability will be an important asset for SWAMSI (Shallow-Water Autonomous Mine Sensing Initiative), BMC (Broadband Mine Classification), LFBB (Low Frequency BroadBand) and the SERDP UXO (Strategic Environmental Research and Development Program, UneXploded Ordnance) Thrust Area, since it is now possible to generate acoustic color data sets for many objects and environmental scenarios. The scientific impact will be all the new modeling techniques developed, which should be useful in other DoD programs as well as in the general structural acoustics community.

In FY04 and FY05 Dr. Burnett researched the commercial FE software and decided to use the code Comsol Multiphysics (then called Femlab) for modeling acoustic scattering from objects in shallow water. It appeared to have the essential physics capabilities and FE techniques for this kind of work, along with a powerful capability permitting users to incorporate their own mathematical expressions, of almost any complexity, in almost any part of the code. It is this latter feature that has made it possible to add the results of the above R\&D to the Comsol software. Now, at the end of FY08, it is clear that the code has fulfilled its promise, performing well beyond initial expectations. It is an excellent platform for both R\&D and production modeling, making possible all the research advances and modeling results described above. Looking ahead to FY09 and beyond, and considering R\&D currently underway, it seems reasonable to expect that the code will be able to handle essentially all the modeling required on these ONR programs.

\section{RELATED PROJECTS}

SERDP (Strategic Environmental Research and Development Program). 
Assessing Sonar Performance Against Underwater UXO. Apply littoral target scattering modeling tools described herein to underwater UXO, focusing on characterization and remediation of UXOcontaminated environments.

\section{REFERENCES}

[1] Burnett, D. S., Sammelmann, G. S., Lee, K. W., "Finite-element modeling of acoustic scattering from objects in shallow water," ONR 2007 Annual Report.

[2] Burnett, D. S., "High-fidelity, high-speed 3-D finite-element modeling of acoustic scattering from littoral targets," ONR Annual Review, AUVSI, Orlando, FL, Feb. 14, 2008.

[3] Baik, Kyungmin, “Acoustical Scattering from Cylinders and Other Objects: Short-Pulse Signatures, Bistatic Synthetic Aperture and Holographic Imaging, and Interfacial Scattering Contributions" (Ph.D. dissertation, Washington State University, Pullman, WA, 2008), p. 266. 